\title{
A Computational Modeling Reveals That Strength of Inhibitory Input, E/I Balance, and Distance of Excitatory Input Modulate Thalamocortical Bursting Properties
}

\author{
Sanggeon Park ${ }^{1,2,3,4}$, Jeong-Woo Sohn ${ }^{1,2}$, Jeiwon Cho ${ }^{1,2 *}$ and Yeowool Huh ${ }^{1,2 *}$ \\ ${ }^{1}$ Department of Medical Science, College of Medicine, Catholic Kwandong University, Gangneung 25601, ${ }^{2}$ Translational \\ Brain Research Center, Catholic Kwandong University International St. Mary's Hospital, Incheon 22711, ${ }^{3}$ Department of \\ Neuroscience, University of Science \& Technology, Daejeon 34113, ${ }^{4}$ Center for Neuroscience, Korea Institute of Science and \\ Technology, Seoul 02792, Korea
}

The thalamus is a brain structure known to modulate sensory information before relaying to the cortex. The unique ability of a thalamocortical (TC) neuron to switch between the high frequency burst firing and single spike tonic firing has been implicated to have a key role in sensory modulation including pain. Of the two firing modes, burst firing, especially maintaining certain burst firing properties, was suggested to be critical in controlling nociceptive behaviors. Therefore, understanding the factors that influence burst firing properties would offer important insight into understanding sensory modulation. Using computational modeling, we investigated how the balance of excitatory and inhibitory inputs into a TC neuron influence TC bursting properties. We found that intensity of inhibitory inputs and the timing of excitatory input delivery control the dynamics of bursting properties. Then, to reflect a more realistic model, excitatory inputs delivered at different dendritic locations-proximal, intermediate, or distal-of a TC neuron were also investigated. Interestingly, excitatory input delivered into a distal dendrite, despite the furthest distance, had the strongest influence in shaping burst firing properties, suggesting that not all inputs equally contribute to modulating TC bursting properties. Overall, the results provide computational insights in understanding the detailed mechanism of the factors influencing temporal pattern of thalamic bursts.

Key words: Computational biology, Thalamus, Sensory gating, T-Type Calcium Channels

\section{INTRODUCTION}

Thalamocortical (TC) neurons which relay sensory information from the spinal cord to the cortex are hypothesized to play important roles in sensory signal modulation [1-3]. Ability of a TC neuron to switch between tonic and burst firing modes have been

Received September 4, 2019, Revised October 26, 2019,

Accepted October 27, 2019

* To whom correspondence should be addressed. Jeiwon Cho, TEL: 82-32-280-6515, FAX: 82-32-280-6510

e-mail:jelectro21@gmail.com

Yeowool Huh, TEL: 82-32-280-6526, FAX: 82-32-280-6510

e-mail:huh06@cku.ac.kr suggested to be the key mechanism of sensory modulation [47]. Based on the arousal state dependent prevalence of each firing mode, tonic firing is suggested to relay peripheral sensory signal to the cortex during the awake state $[4,8]$, while burst firing is assumed to block sensory information from being transferred to the cortex $[8,9]$.

Many studies demonstrate the importance of TC burst firing in pain [10-14]. Recent studies suggested that maintaining certain TC bursting properties would be important for pain modulation. In pathological pain models, spinal cord injury (SCI), the properties of bursts significantly differed from those of the sham control $[10,11]$. Thalamic activities of animals with pathological pain had higher burst firing frequency, but shorter burst length, fewer burst spikes composing a burst, and longer interval between burst spikes
Copyright $\odot$ Experimental Neurobiology 2019.

www.enjournal.org
This is an Open Access article distributed under the terms of the Creative Commons Attribution Non-Commercial License (http://creativecommons.org/licenses/by-nc/4.0) which permits unrestricted non-commercial use, distribution, and reproduction in any medium, provided the original work is properly cited. 
(Intra-burst-interval, IntraBI). Even in non-pathological states, TC neuronal single unit recording and electrical stimulation studies showed that changing dynamics of TC burst firing properties differentially modulate nociceptive behaviors [12, 13]. Especially the length of IntraBI and the number of burst spikes within a burst were demonstrated to be important burst features influencing the degree that nociceptive behavior were modulated [1215]. Firing mode of TC neurons are determined by the balance of excitatory and inhibitory inputs within the TC circuit $[16,17]$. The reticular thalamus provides inhibitory inputs, while the cortex and spinothalamic tract provide excitatory inputs to TC neurons [2]. However, how the balance between excitatory and inhibitory inputs influence TC burst firing properties has not yet been investigated. Also, excitatory and inhibitory inputs are known to form synapses at different locations: while different types of excitatory inputs form different types of synapses along the dendrites of a TC neuron, inhibitory inputs mostly synapse onto cell bodies [18]. Therefore, studying the factors that influence TC bursting properties would provide valuable information in understanding how nociceptive information is modulated at thalamic level and is expected to further provide clues to understanding general sensory gating mechanisms.

We attempted to dissociate the factors that influence TC bursting properties using computational modeling. We first investigated the sole effect of synaptic inhibition on the temporal patterning of TC bursting, then how the timing and intensity of excitatory input delivered into the soma under weak or strong inhibitory input modulates TC bursting properties. Lastly, we investigated the relationship between excitatory inputs delivered at different dendritic locations and TC bursting properties.

\section{MATERIALS AND METHODS}

\section{Thalamocortical NEURON model}

Neural modeling software NEURON 7.2 [19], adopted from Destexhe et al. [17], was used for all simulations on a HP Z420 multi-processing workstation. The model included passive, voltage-dependent sodium, voltage-dependent potassium, and T-type $\mathrm{Ca}^{2+}$ channels. The maximal conductance of $\mathrm{g}_{\mathrm{Na}}\left(100 \mathrm{mS} / \mathrm{cm}^{2}\right)$ and $\mathrm{gK}\left(100 \mathrm{mS} / \mathrm{cm}^{2}\right)$ were uniformly distributed in the model. However, $\mathrm{P}_{\mathrm{Ca}}$ (the maximum permeability of the membrane to $\mathrm{Ca}^{2+}$ ions) was modeled as follows to reflect unequal distribution of Ttype $\mathrm{Ca}^{2+}$ channels with distance from the soma [17].

For $\mathrm{P}_{\mathrm{Ca}}[17]$,

$$
\left\{\begin{array}{c}
g_{\text {somatic and proximal dendritic }}=1.7 e^{\wedge}(-5) \mathrm{cm} / \mathrm{s} \\
g_{\text {medial and distal dendritic }}=8.5 e^{\wedge}(-5) \mathrm{cm} / \mathrm{s}
\end{array}\right.
$$

\section{Model simulation condition}

To investigate the sole effect of inhibitory input in modulating TC bursting patterns, inhibitory post synaptic current (IPSC) intensity $-0 \mathrm{nA} \sim-0.5 \mathrm{nA}$ in $0.05 \mathrm{nA}$ step size and duration $0 \mathrm{~ms} \sim 200$ $\mathrm{ms}$ in $20 \mathrm{~ms}$ increments was delivered to the soma.

Since a real TC neuron receives both excitatory and inhibitory inputs, we investigated how the timing of excitatory post synaptic current (EPSC), in the presence of IPSC, affect temporal pattern of TC bursting. In this model, EPSC was injected into the soma at different time points during two different IPSC intensity conditions: strong (-0.3 nA) and weak (-0.1 nA). IPSC intensities for strong and weak conditions were chosen to be within the range of IPSCs that induced low-threshold spikes (LTS) (-0.1 to -0.8 nA) in patch clamp studies $[7,17,20,21]$.

We also investigated how synaptic location of excitatory post synaptic current delivered affect TC bursting properties. Three EPSC input locations along the dendrite (proximal $=19 \mu \mathrm{m}$, intermediate $=52 \mu \mathrm{m}$, and distal $=247 \mu \mathrm{m}$ depending on the distance from the soma) were chosen based on the study by Liu et al. (1995) [18]. Other conditions were kept the same as the excitatory and inhibitory balance modeling used in this study.

\section{Data analysis}

To investigate how bursting properties are modulated by the balance of inhibitory and excitatory inputs, changes in the 1st IntraBI (decrease/increase), burst onset timing (advancement/delay), and the number of burst spikes relative to those of the reference, were plotted as a function of EPSC input timing. These analysis were adapted from the spike time response curve (STRC) analysis which measures how a stimulus affects the timing of a neuron's action potential $[22,23]$.

The effect of different EPSC intensities was also investigated using the same analysis. We used MATLAB fit function to fit the results. The following formula was used for an exponential fit

$$
\operatorname{axexp}\left(b^{*} x\right)
$$

Each exponential fit was verified using the root-mean-square error (RMSE).

The effect of EPSC delivered at different dendritic locations in changing burst properties was also analyzed by plotting respective changes in the 1st IntraBI length, burst onset timing, and number of burst spikes as a function of EPSC input timing.

All data were analyzed using custom-made procedures in MATLAB (version R2014b). 


\section{RESULTS}

Inhibitory input controls temporal pattern of thalamocortical burst firing

A multi-compartment TC NEURON model [17, 19, 24], illustrated in Fig. 1A, was used to investigate the relationship between variations in IPSCs and TC burst spiking patterns. After confirming that IPSC (current: $-0.3 \mathrm{nA}$, duration: $200 \mathrm{~ms}$ ) delivered into the soma of the TC neuron model triggers LTS in a similar pattern to those recorded in-vitro (Fig. 1B) [17, 20], we investigated how IPSC intensity ( $0 \mathrm{nA}$ to $-0.5 \mathrm{nA}$ in $0.05 \mathrm{nA}$ step size) and duration (0 ms to $200 \mathrm{~ms}$ ) affect the length of the1st IntraBI and the number of burst spikes composing a burst. Length of the 1st IntraBI decreased with increasing IPSC amplitude and duration (Fig. 1C). As IPSC amplitude increased, the minimum IPSC duration to trigger LTS became shorter. Similarly, longer IPSC duration low-
A) Thalamocortical

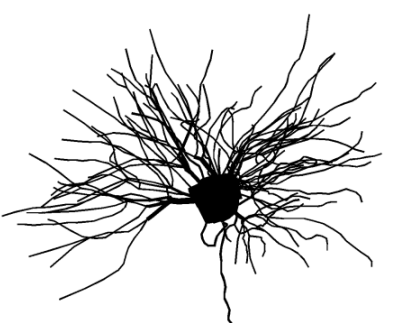

B) Low-threshold

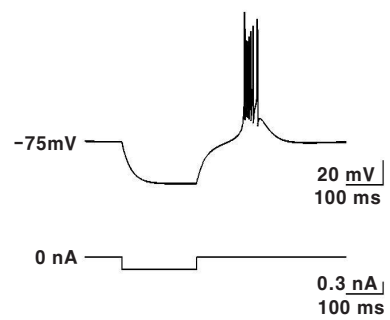

C) $1^{\text {st }}$ Intra Burst Interval

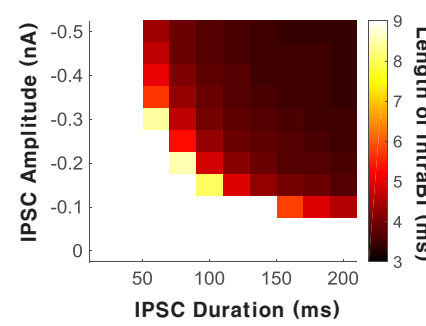

D) Low-threshold $\mathrm{Ca}^{2+}$ Burst Spikes Number

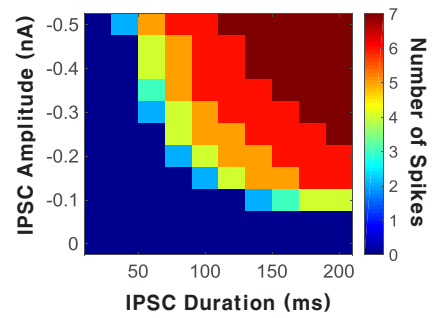

Fig. 1. Computational thalamocortical neuron model and burst firing properties modulated by IPSC characteristics. (A) Thalamocortical NEURON model used in the study (200 multi-compartments structure). (B) Example of a Low-threshold $\mathrm{Ca}^{2+}$ burst spikes voltage trace (top) and a current trace (bottom) delivered at the soma. (C) Heat map depicting the relationship between the length of 1st IntraBI and IPSC. (D) Heat map showing the relationship between the number of burst spikes composing a LTS burst and IPSC.

A) Strong inhibitory LTS

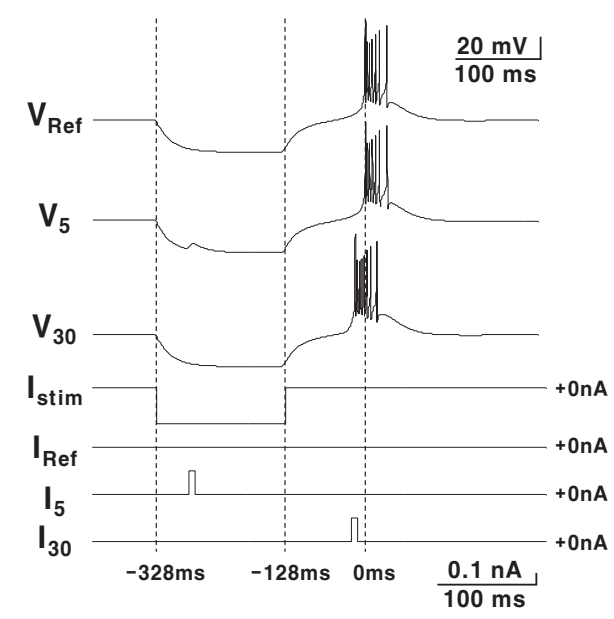

C)

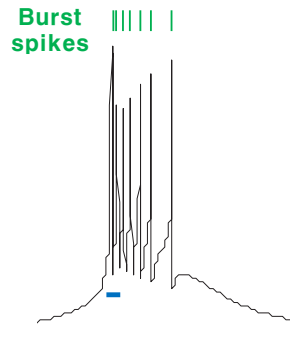

Burst Spike
D)

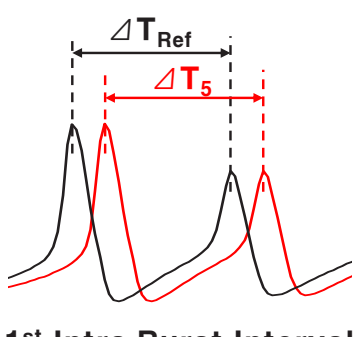

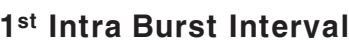

B)

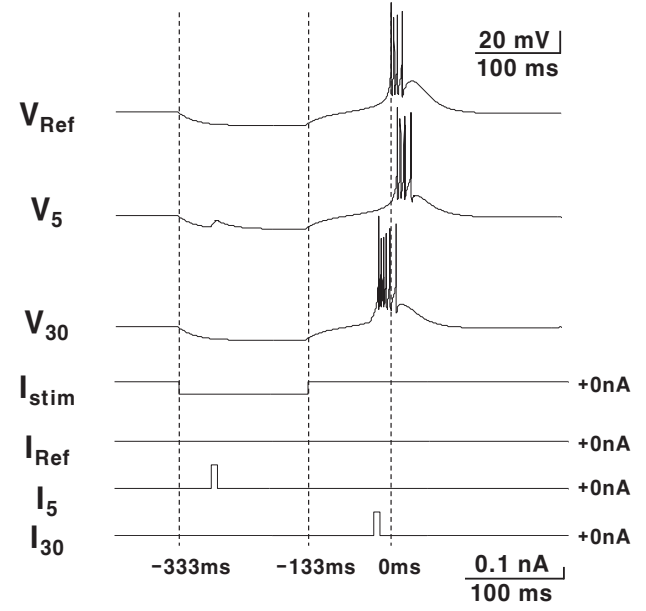

E)

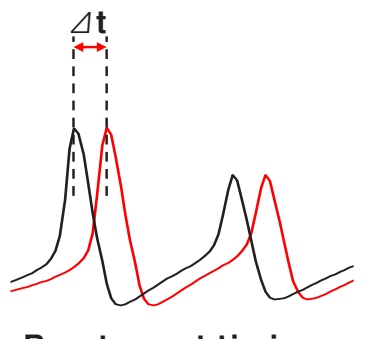

Fig. 2. Simulation conditions for the excitatory and inhibitory balance on burst properties. (A) Sample voltage traces of strong IPSC condition. (B) Sample voltage traces of weak IPSC condition. (A, B) $\mathrm{V}_{\text {ref }}$ only IPSC current $\left(\mathrm{I}_{\text {stim }}\right) . \mathrm{V}_{5}$ and $\mathrm{V}_{30}$ : EPSC delivered at the fifth $\left(\mathrm{I}_{5}\right)$ and the thirtieth $\left(I_{30}\right)$ time point, respectively. Vertical dotted lines and the number below, from left to right, sequentially indicate the time points for the start and end of IPSC, and $\mathrm{V}_{\text {ref }}$ burst onset. (C) Example of a burst. Vertical green bars indicate when burst spikes occurred. (D) $\Delta T_{\text {Ref: }}$ 1st IntraBI length of $V_{\text {ref }}$. $\Delta \mathrm{T}_{5}: 1$ st IntraBI length of $\mathrm{V}_{5}$. (E) $\Delta t$ indicate the shift in the first burst spike onset timing by an excitatory stimulus. 
ered the minimum IPSC amplitude to trigger LTS. The number of burst spikes comprising a burst had a tendency to increase with greater IPSC amplitude and longer IPSC duration (Fig. 1D). Overall, results showed that variations in IPSC amplitude and duration contribute to determining the length of the 1st IntraBI and the number of burst spikes within a burst.

\section{Timing of excitatory input modulates TC bursting properties}

Neurons functioning within a network receive various excitatory and inhibitory inputs [18]. A TC neuron also integrates both excitatory and inhibitory inputs incoming at different time points, thus, how the balance of excitatory/inhibitory inputs into a TC neuron modulates the TC burst firing dynamics was investigated. Assumptions and conditions of the simulation used for the study are depicted in Fig. 2. All inputs, both IPSC and EPSC, were injected into the soma for simplification. Two conditions of IPSCs $(200$ ms, $-0.3 \mathrm{nA}$ (strong) or $-0.1 \mathrm{nA}$ (weak)) were used in the study to investigate how the magnitude of IPSCs influence TC bursting properties. Both the strong and weak IPSC were able to trigger
LTS (Fig. 2A and 2B, $\mathrm{V}_{\text {Ref }}$ ). EPSCs (10 ms, 0.05 0.25 nA in $0.05 \mathrm{nA}$ increments) were injected at different time points starting from when IPSC was delivered until when the burst occurred in the reference to investigate the relationship between EPSC delivery timing and TC bursting properties. Specifically, we examined how EPSC injection timing and amplitude affect the length of the 1st IntraBI, onset timing of bursts, and number of burst spikes during both the weak and strong IPSC conditions. A burst is composed of burst spikes as shown in Fig. 2C. The 1st IntraBI was defined by the time difference between the 2 nd and 1st burst spikes within a burst, as shown in Fig. 2C and 2D $(\Delta \mathrm{T}=2 \mathrm{nd}$ - 1st burst spike occurrence time). Fig. 2D shows a sample of a 1st IntraBI length calculated for a reference (black), which had no EPSC injection, and an EPSC injected at time point 5 (red). In addition, changes in the burst onset timing due to EPSC injection at different time points was calculated by the time difference between the first burst spike occurrence of a reference (black) and an EPSC injection condition (red) ( $\Delta \mathrm{t}=1$ st burst spike occurrence time of Ref -1 st burst spike occurrence time of EPSC injection, Fig. 2C and 2E).

As predicted, the timing of EPSC delivery influenced TC burst
A)

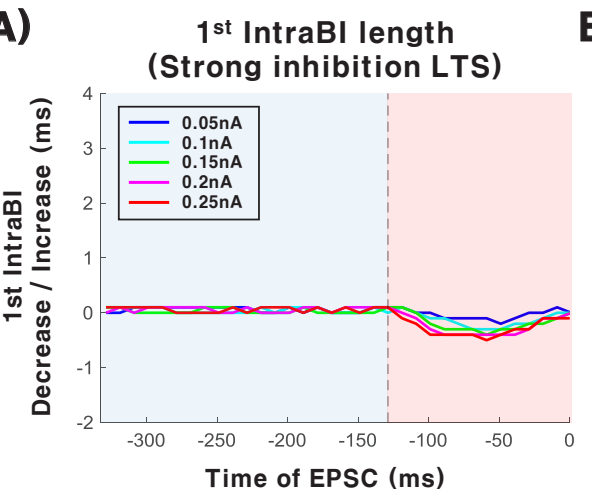

D)

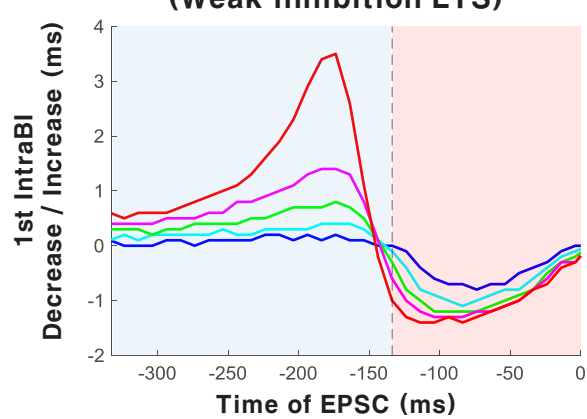

B)

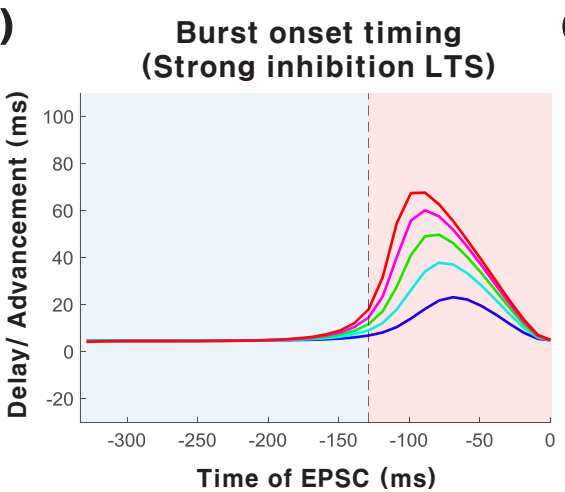

E)

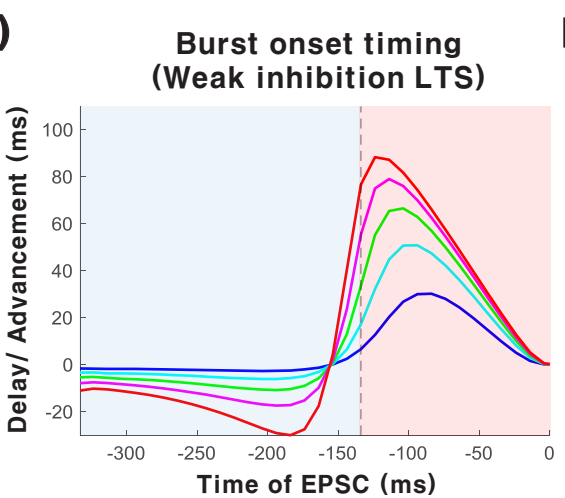

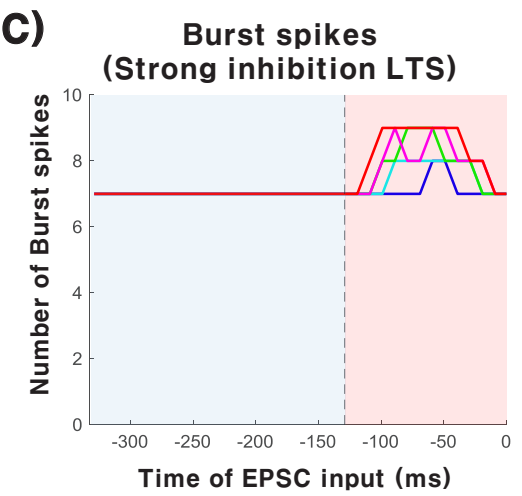

F)
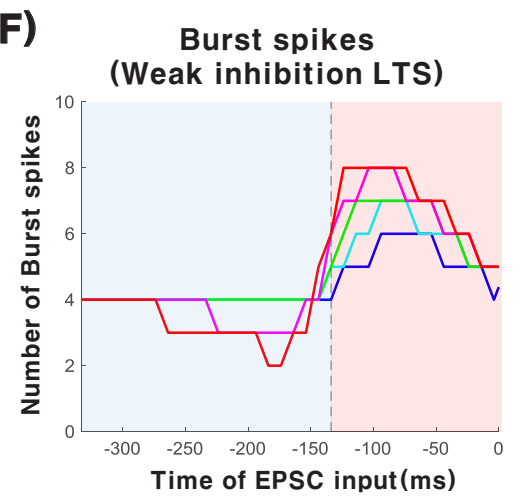

Fig. 3. Thalamocortical bursting properties modulated by excitatory inputs delivered during strong or weak inhibition. (A) Changes in the length of 1 st IntraBI under strong inhibition. (B) Spike-time response curve (STRC) under strong inhibition. (C) Changes in the number of burst spikes under strong inhibition. (D) Changes in the length of 1st IntraBI under weak inhibition. (E) STRC under weak inhibition. (F) Changes in the number of burst spikes under weak inhibition. (A F) Vertical dotted line: the time point when IPSC delivery stopped. 
firing properties: the length of the 1st IntraBI, timing of burst onset, and number of burst spikes within a burst. However, the aspect of burst property changes was different for strong and weak IPSC conditions. Under the strong IPSC condition, EPSC injected after IPSC injection had a tendency to decrease the length of the 1st IntraBI (Fig. 3A). Value below zero indicates decrease in the length of IntraBI compared to the reference, and vice versa, in this plot. Length of the 1st IntraBI got shorter with greater EPSC injected after the time point of IPSC injection (Fig. 3A, pink area). Burst onset timing was also influenced by the timing of EPSC delivery. EPSC injected after release from IPSC injection had a tendency to advance burst onset timing (Fig. 3B, pink area). In this plot, value above zero indicates advancement while value below zero indicates delay of burst onset. Burst occurrence advancement was positively correlated with the magnitude of EPSC. The number of burst spikes compositing a burst was also increased by EPSC injected after IPSC release (Fig. 3C, pink area). Under strong IPSC condition, EPSC injection could only decrease the length of the 1st IntraBI, advance the burst onset timing, or increase the number of burst spikes. Interestingly, no matter how strong the EPSC becomes, modulation of burst properties occurred only in one direction under the strong IPSC condition. We tested up to $0.7 \mathrm{nA}$ EPSC in $0.05 \mathrm{nA}$ steps, and found that no spikes occurred when $0.65 \mathrm{nA}$ EPSC was delivered and only tonic spikes occurred with $0.7 \mathrm{nA}$ EPSC injection. However, under the weak the IPSC condition, EPSC injection was able to modulate TC bursting properties bi-directionally. EPSC injection decreased or increased the 1st IntraBI length, delayed or advanced the burst onset timing, and increased or decreased the number of burst spikes depending on the time EPSC was delivered (Fig. 3D F). EPSC delivered before IPSC release (light blue area) increased the length of the 1st IntraBI, delayed burst onset, and decreased the number of burst spikes, which were trends not seen in the strong inhibition condition. EPSC delivered after IPSC release (pink area) decreased the length of the 1st IntraBI, advanced burst onset, and increased the number of burst spikes, in similar trends as observed in the strong inhibition condition, but the range that burst properties were modulated was greater under the weak IPSC condition than under the strong IPSC condition.

\section{Intensity of excitatory input affects bursting properties}

The relationship between the amplitude of EPSC and bursting properties was investigated in more detail by plotting the maximum decrease/increase of the 1st IntraBI length, maximum advance/delay of burst onset timing, and maximum increase/ decrease in the number of burst spikes over five different EPSC amplitudes (0.05 0.25 nA in 0.05 increments). Decrease in the maximum length of the 1st IntraBI occurred in both strong and weak inhibition conditions, with greater EPSC amplitude triggering greater decrease in the 1st IntraBI length (Fig. 4A). Maximum decrease of the 1st IntraBI length was fitted using an exponential

A)

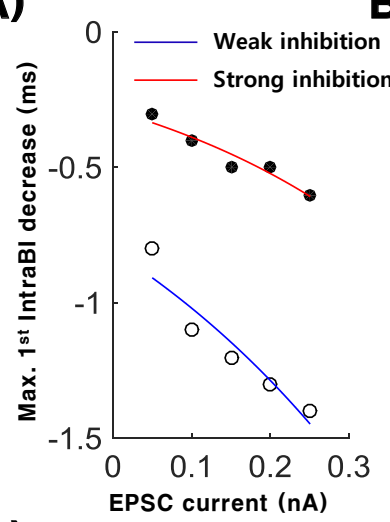

C)
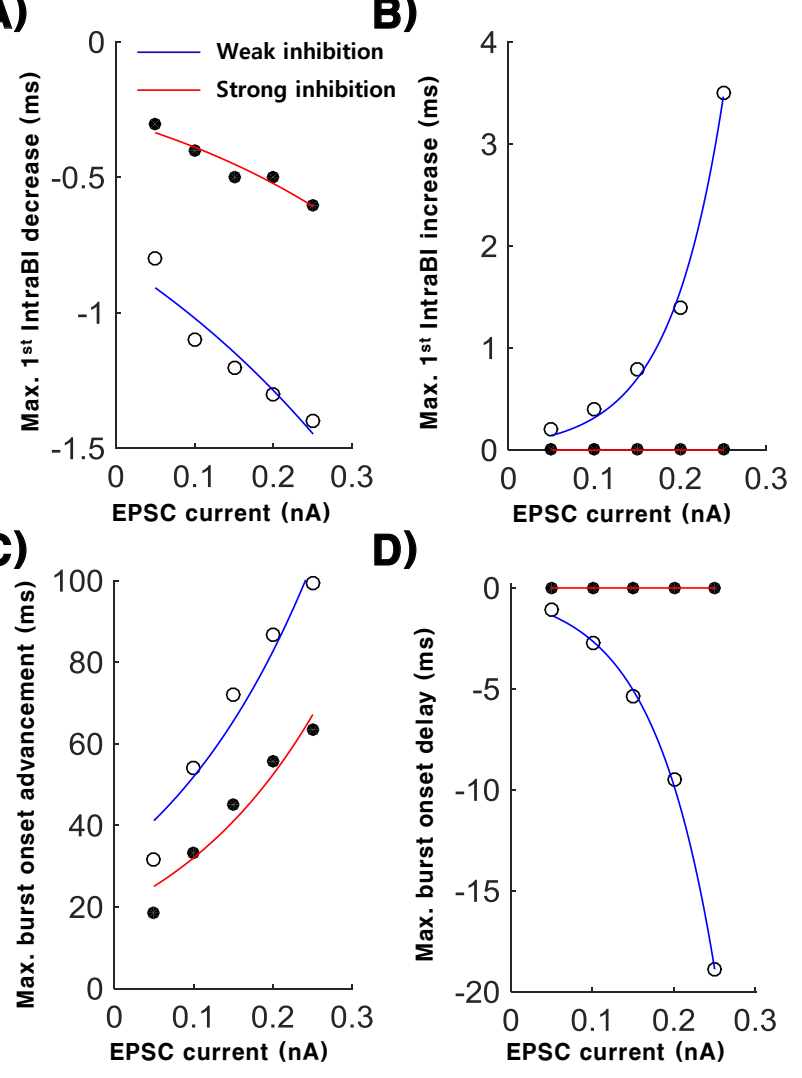

D)

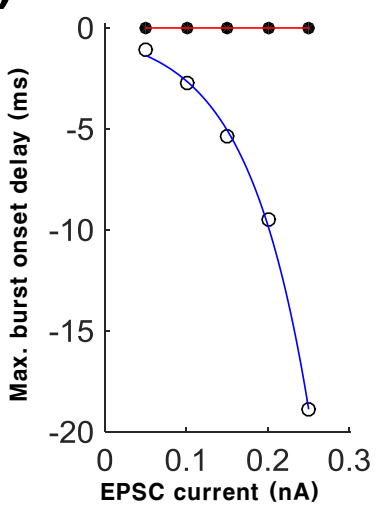

E)

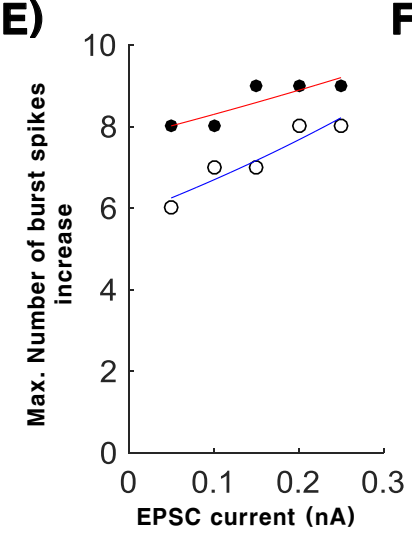

F)

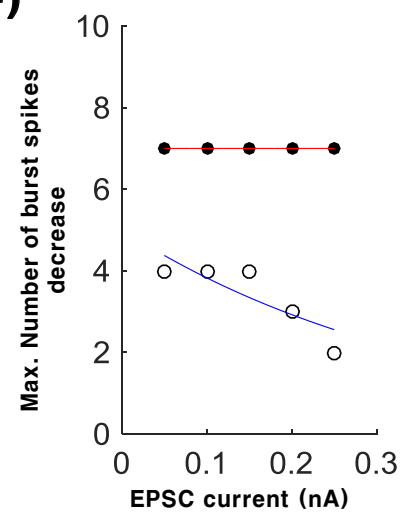

Fig. 4. Magnitude of excitatory post synaptic input current-dependent control of burst spike temporal dynamics. (A) Maximum 1st IntraBI length decrease depends on increasing EPSC stimulation current. (B) Maximum 1st IntraBI length increase depends on increasing EPSC stimulation current. (C) Maximum spike onset advancement depends on EPSC magnitude. (D) Maximun spike onset delay depends on EPSC magnitude. (E) Maximum number of burst spikes increase by EPSC. (F) Maximum number of burst spikes decrease by EPSC. 
A) Thalamocortical Neuron

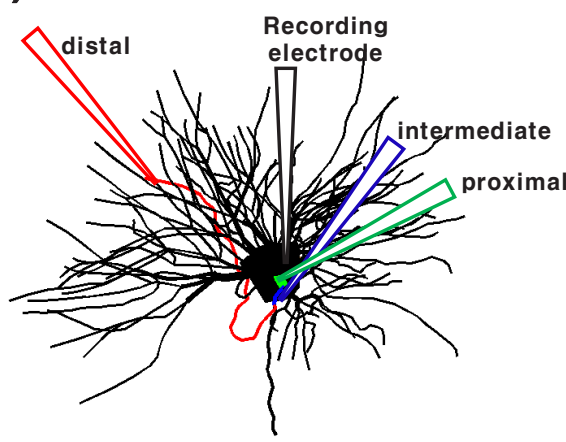

1 st IntraBI response curve
(Strong Inhibition LTS)

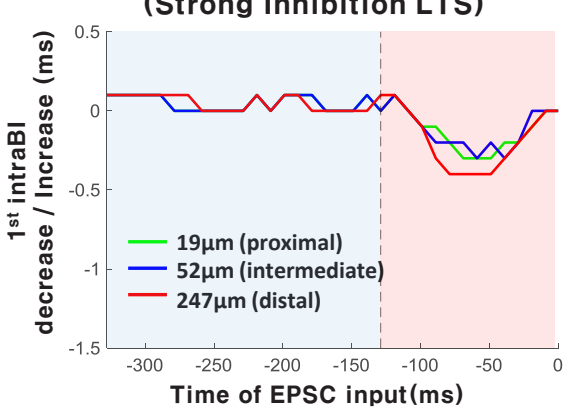

$1^{\text {st }}$ IntraBI response curve
(Weak Inhibition LTS)

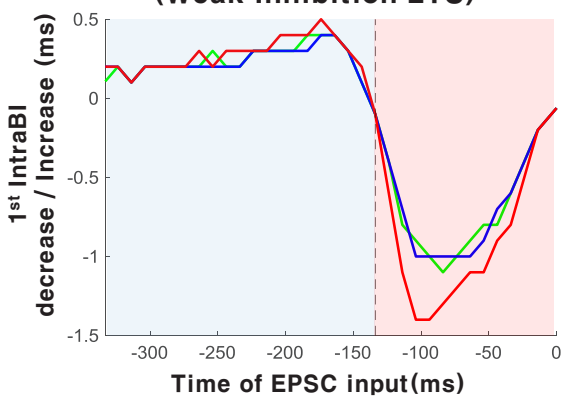

B)

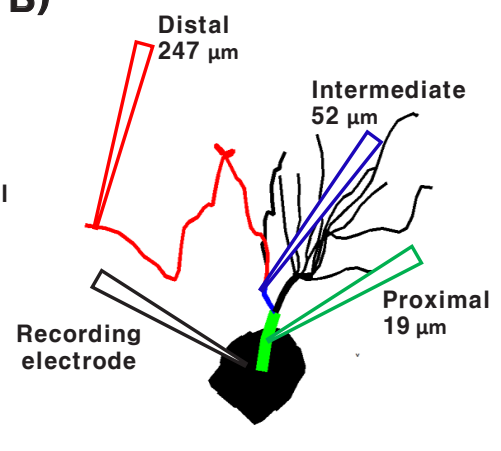

E)

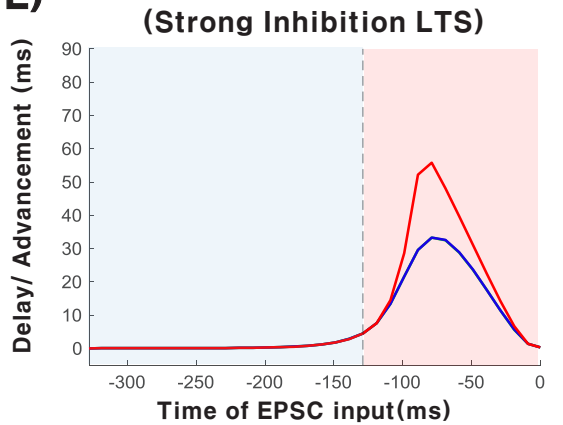

H)

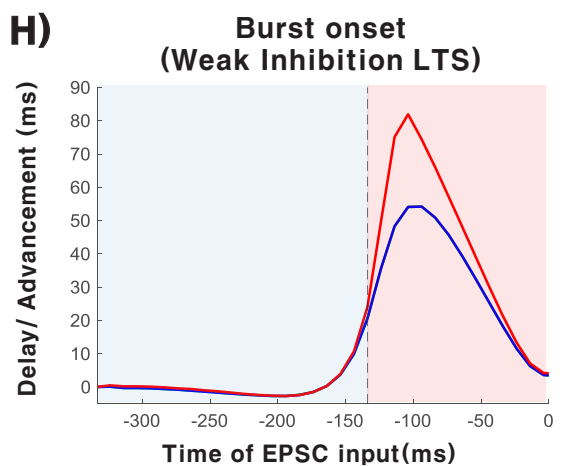

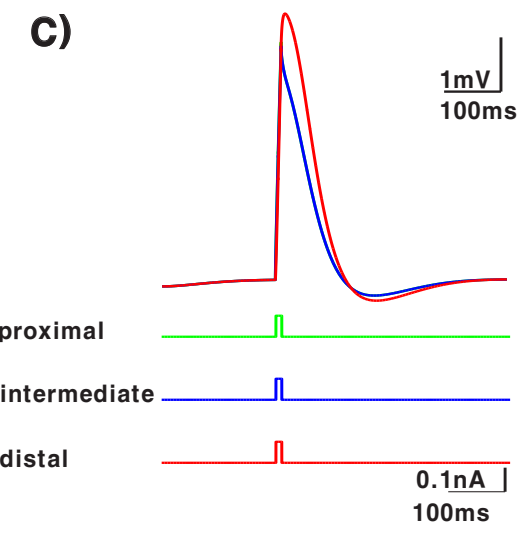

F) Intra Burst spikes

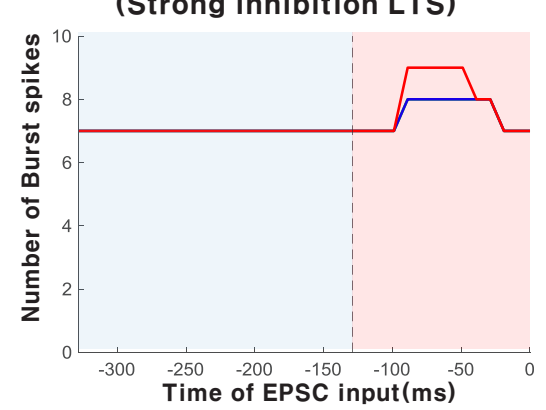

l)
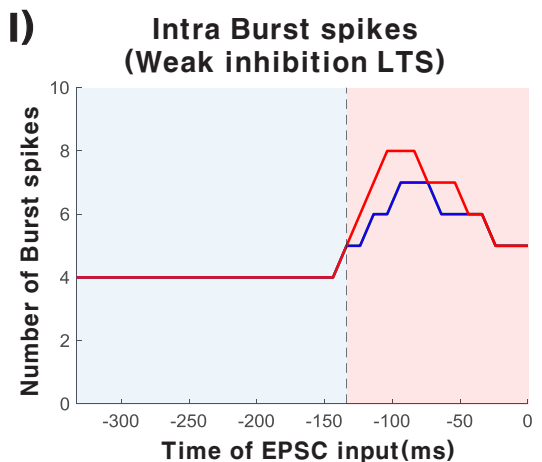

Fig. 5. Synaptic input location dependent modulation of bursting properties. (A) Stimulation and recording locations used in the experiment. (B) Detailed depiction of the stimulated dendrite. (C) Voltage traces of the model thalamocortical neuron by EPSCs injected at three different dendritic locations. (D) Length of the 1st IntraBI under strong inhibition. (E) Burst onset timing under strong inhibition. (F) Number of burst spikes under strong inhibition. (G) Length of the 1st IntraBI under weak inhibition. (H) Burst onset timing under weak inhibition. (I) Number of burst spikes under weak inhibition. (D I) Vertical dotted line: the time point when IPSC delivery stopped.

function (weak inhibition: $\mathrm{a}=-0.299, \mathrm{~b}=2.965$ and RMSE $=0.038$; strong inhibition: $\mathrm{a}=-0.089, \mathrm{~b}=2.327$ and $\mathrm{RMSE}=0.088$ ). Increase in the maximum 1st IntraBI length, on the other hand, only occurred in the weak inhibition condition (Fig. 4B, blue line). Maximum length of the 1st IntraBI increased exponentially with increasing intensity of EPSC under the weak inhibition condition (Fig. 4A blue line, $\mathrm{a}=0.064, \mathrm{~b}=15.930$, RMSE $=0.125$ ).

Investigation of the relationship between EPSC magnitudes and maximum advancement or delay of burst onset timing (Fig. 4C and 4D) revealed that maximum advancement or delay got greater with increasing EPSC injected. Advancement of maximum burst onset timing occurred, by EPSC injected after IPSC release, in both weak and strong inhibition conditions, with increasing EPSC amplitude causing greater advancement (Fig. 4C, weak inhibition: $a=32.640, b=4.644$ and RMSE $=7.646$; strong inhibition: $a=19.600$, $\mathrm{b}=4.919$ and RMSE $=5.411$ ). However, maximum delay of burst onset timing only occurred in the weak inhibition condition (Fig. $4 \mathrm{D})$. In the weak inhibition condition, delay of burst onset timing 
Table 1. Average burst firing properties under weak and strong inhibition conditions

\begin{tabular}{|c|c|c|c|c|c|c|c|}
\hline \multicolumn{4}{|c|}{ Weak inhibition } & \multicolumn{4}{|c|}{ Strong inhibition } \\
\hline EPSC location & $\begin{array}{l}\text { 1st IntraBI } \\
\text { (ms) }\end{array}$ & $\begin{array}{c}\text { Last IntraBI } \\
\text { (ms) }\end{array}$ & $\begin{array}{l}\text { Number of } \\
\text { burst spike }\end{array}$ & EPSC location & $\begin{array}{c}\text { 1st IntraBI } \\
\text { (ms) }\end{array}$ & $\begin{array}{c}\text { Last IntraBI } \\
\text { (ms) }\end{array}$ & $\begin{array}{l}\text { Number of } \\
\text { burst spike }\end{array}$ \\
\hline Proximal & $4.40 \pm 0.07$ & $7.20 \pm 0.28$ & $4.71 \pm 0.15$ & Proximal & $3.38 \pm 0.02$ & $10.45 \pm 0.34$ & $7.17 \pm 0.06$ \\
\hline Intermediate & $4.34 \pm 0.10$ & $7.20 \pm 0.35$ & $4.98 \pm 0.22$ & Intermediate & $3.35 \pm 0.03$ & $10.16 \pm 0.44$ & $7.20 \pm 0.06$ \\
\hline Distal & $4.35 \pm 0.09$ & $7.19 \pm 0.33$ & $4.90 \pm 0.20$ & Distal & $3.37 \pm 0.02$ & $10.25 \pm 0.42$ & $7.17 \pm 0.06$ \\
\hline
\end{tabular}

got greater with increasing EPSC amplitude $(\mathrm{a}=19.600, \mathrm{~b}=4.919$ and RMSE=5.411).

The number of burst spikes composing a burst also changed depending on the magnitude of EPSC delivered (Fig. 4E and 4F). The strong inhibition condition triggered a burst to have more burst spikes (7 burst spikes) compared to that of the weak inhibition condition (4 burst spikes) when no EPSC was delivered. The maximum number of burst spikes increased with increasing EPSC for both weak and strong inhibition conditions (Fig. 4E, weak inhibition: $\mathrm{a}=5.837, \mathrm{~b}=1.368$ and $\mathrm{RMSE}=0.336$; strong inhibition: $\mathrm{a}=7.746, \mathrm{~b}=0.689$ and RMSE $=0.322$ ). The modulation range was shown to be greater for the weak inhibition condition (from 4 to 8 burst spikes) than the strong inhibition condition (from 7 to 9 burst spikes). The maximum number of burst spike decrease, in contrast, only occurred in the weak inhibition condition, in which more burst spike number decreased with greater EPSC injected (Fig. 4F blue line, $\mathrm{a}=5.837, \mathrm{~b}=1.368$ and $\mathrm{RMSE}=0.553$ ).

Results show that TC burst firing properties had more modulation flexibility under the weak IPSC condition compared to the strong IPSC condition. Not only were bursting properties modulated bi-directionally, but magnitude of bursting property changes were also greater under the weak IPSC condition.

\section{Excitatory input delivered at different dendritic locations of a thalamocortical neuron differentially modulate bursting properties}

Different types of synapses with distinct inputs are known to be present along the dendrites of a TC neuron, of which the distribution density differs with distance [18]. Therefore, we investigated how EPSC delivered at different synaptic input locations differentially affect burst firing properties. Dendritic locations at $19 \mu \mathrm{m}, 52$ $\mu \mathrm{m}$, and $247 \mu \mathrm{m}$ from the soma were used to represent proximal, intermediate, and distal dendrite, respectively (Fig. 5A and 5B). Fig. $5 \mathrm{~B}$ illustrates a more detailed image of the dendrite used in this simulation. Other than the EPSC delivered at different TC dendritic locations, same simulation and analysis paradigm described in Fig. 2 were used. Sample of TC neuron membrane voltage traces triggered by injecting EPSC $(0.1 \mathrm{nA})$ at different dendritic loca- tions are plotted in Fig. 5C. The main objective was to investigate the difference effects of EPSC injected at different dendritic locations, so EPSC amplitude was fixed. Interestingly, voltage traces show that excitatory input delivered at the distal dendrite, despite the furthest distance from the soma, triggered the biggest peak depolarization of membrane potentials (Fig. 5C, red). Excitatory input delivered into the proximal and intermediate dendrites triggered similar levels of peak membrane depolarization and had a slower decay of depolarization compared to the distal stimulation (Fig. 5C).

Next, we investigated the modulating influence of EPSC delivered at different dendritic locations on burst firing properties. Variations in the 1st IntraBI length, burst onset timing, and the number of burst spikes were examined under strong and weak IPSC conditions. EPSC injection ( $10 \mathrm{~ms}, 0.1 \mathrm{nA})$ at different dendritic locations basically had similar influence on bursting properties as EPSCs injected into the soma (Fig. 5D I). Average values of the bursting properties simulated in our conditions are summarized in Table 1. An interesting point to note is that EPSC delivered into the distal dendrite had the greatest influence in modulating burst properties than EPSC injection at other locations, despite the furthest distance from the soma. This implies that corticothalamic inputs in to a TC neuron may have the strongest influence in modulating TC burst firing properties, because majority of the synapses present in the distal dendrite are RS-type (round vesicles, small), which are mainly corticothalamic [18]. Overall, results suggest that different types of synapses present along the dendrite of TC neurons will differentially modulate TC burst firing properties and may further contribute to differential modulation of sensory signals.

\section{DISCUSSION}

Our study evaluated various factors modulating TC burst firing dynamics. Different conditions of inhibitory input, balance between inhibitory and excitatory inputs, and location of dendritic excitatory input all affected bursting properties. The results provide valuable insights into the detailed sensory gating mechanisms 
mediated by thalamic bursts, since studies suggest that maintaining certain bursting properties would be important for controlling nociceptive responses [12-15] and processing complex sensory information $[4,5,7]$.

\section{Inhibitory inputs modulating TC bursting properties}

The major inhibitory input, which is required to enable TC burst firing, is provided by the reticular thalamic nucleus (RT) [2]. Our simulation results showed that the strength of IPSC input affect TC bursting properties. Several factors could affect the strength of IPSC. Synchronized activity of RT neurons could be one factor, since synchronization of RT neurons increase IPSC amplitude on TC neurons [25]. Different synaptic connections RT forms with TC neurons, the 'strong' or 'weak', could be another factor. The 'strong' connection, which has significantly more axonal branching than the 'weak' connection, induces significantly greater IPSC compared to the 'weak' connection [7,26]. Differences in neuronal types in the RT could also be a factor. Neurons that have longer burst, the typical neurons, are likely to induce greater IPSC [27]. Yet another way that IPSC magnitudes could be controlled is through the TC feedback loop. The RT receives feedback from TC neurons. Therefore, as gain from the feedback loop increased, greater IPSC was induced [28]. This means that accumulation of TC activity, as in sustained pain, would lead to the generation of greater IPSCs that would cause TC neurons to bursts with properties that is effective for reducing nociceptive responses. This suggests that dysfunction in TC inhibitory circuit could be the cause of pathological pain conditions, since altered GABAergic signaling causes various brain diseases [29].

Balance of inhibitory and excitatory inputs modulating TC bursting properties

Realistically a TC neuron integrates both excitatory and inhibitory inputs. We investigated how the timing and magnitude of EPSC affects TC bursting properties under two IPSC conditions (weak or strong), and found that modulation range of bursting properties was greater in the weak IPSC condition. Notably, under the weak IPSC condition, bursting properties changed bi-directionally. In contrast, under the strong IPSC inhibition, bursting properties changed only in one direction, regardless of the EPSC magnitude. This result suggest that burst firing properties would have more modulation flexibility under the weak IPSC condition. The weak IPSC condition used in our study may better reflect how bursting properties are modulated in the awake state because activity of RT neurons are desynchronized in the awake state [30], and this alludes that bursting properties are more actively modulated in the awake state.

\section{Excitatory inputs delivered at different dendritic location modulating TC bursting properties}

EPSC delivered at different dendritic locations were investigated, since different type of synapses are reported to be formed along the dendrite of a TC neuron [18]. Surprisingly, EPSC input into the distal dendrite, despite the furthest distance from the soma, triggered the greatest response and also had greatest influence in modulating TC bursting properties compared to EPSC inputs delivered into the intermediate or proximal dendrites. This phenomena, which seemingly defies the cable theory, was likely due to the greatest T-type $\mathrm{Ca}^{2+}$ channel density in distal dendrites of a TC neuron [17]. This results also suggest that corticothalamic input may have the greatest influence in modulating TC bursting dynamics, because corticothalamic inputs mostly synapse onto distal dendrites, whereas the EPSC input coming from the periphery and brainstem mostly synapse onto proximal and intermediate dendrites of a TC neuron [18].

\section{Implications of burst firing properties dynamics}

The thalamus is suggested to be involved in sensory gating, and burst in certain forms may be more effective in reducing sensory information transfer. In case of pain, TC bursts would reduce nociception in two steps: by inhibition of TC activity and by activation of cortical inhibitory neurons. A recent study showed that increasing inhibition in the TC by activating the RT, reduced nociceptive behaviors [31]. Also, TC bursts selectively activated cortical parvalbumin expressing inhibitory interneurons, which reduced nociceptive behaviors when activated [14]. Specific TC bursting properties that are apt to reduce nociception may be in a resonance frequency that are more likely to activate cortical PV interneurons, because neurons respond maximally to their natural resonance frequencies [32,33].

Modulation of TC bursting properties are also expected to be important for processing complex sensory signals. Not only are bursts suggested to make signal transfer more reliable $[5,6,34]$, different TC bursting patterns could preferentially activate different types of neurons by resonance $[32,33]$. A study also suggested that TC bursts function in multiplexed signal processing [35]. Therefore, the results of our study, which elucidated the factors that contribute to modulating TC bursting properties, will further provide basis for better understanding the mechanisms underlying complex sensory modulation.

For simplicity we have only considered passive, voltage-dependent sodium, voltage-dependent potassium, and T-type $\mathrm{Ca}^{2+}$ channels in this study. It would, however, be interesting to investigate how other channels such as the $\mathrm{Ca}^{2+}$-activated $\mathrm{K}$ - and $\mathrm{Cl}$ - channels expressed in TC neurons, which were shown to have roles in 
modulating nociception [36-38], influence TC bursting dynamics in future studies.

Overall, our study provide valuable information about the factors that modulate bursting dynamics of a TC neuron.

\section{ACKNOWLEDGEMENTS}

This research was funded by the Ministry of Science ICT through the National Research Foundation of Korea (NRF) grants: Brain Science Research Program (NRF-2015M3C7A1028392, NRF-2016M3C7A1904986, NRF-2018M3C7A1024736) and NRF-2018R1C1B6002210.

\section{REFERENCES}

1. McCormick DA, Bal T (1994) Sensory gating mechanisms of the thalamus. Curr Opin Neurobiol 4:550-556.

2. Jones EG (1985) The thalamus. Plenum Press, New York, NY.

3. Dostrovsky JO (2000) Role of thalamus in pain. Prog Brain Res 129:245-257.

4. Sherman SM (2001) Tonic and burst firing: dual modes of thalamocortical relay. Trends Neurosci 24:122-126.

5. Krahe R, Gabbiani F (2004) Burst firing in sensory systems. Nat Rev Neurosci 5:13-23.

6. Lisman JE (1997) Bursts as a unit of neural information: making unreliable synapses reliable. Trends Neurosci 20:38-43.

7. Zhan XJ, Cox CL, Sherman SM (2000) Dendritic depolarization efficiently attenuates low-threshold calcium spikes in thalamic relay cells. J Neurosci 20:3909-3914.

8. Livingstone MS, Hubel DH (1981) Effects of sleep and arousal on the processing of visual information in the cat. Nature 291:554-561.

9. Steriade M, McCormick DA, Sejnowski TJ (1993) Thalamocortical oscillations in the sleeping and aroused brain. Science 262:679-685.

10. Hains BC, Saab CY, Waxman SG (2006) Alterations in burst firing of thalamic VPL neurons and reversal by $\mathrm{Na}(\mathrm{v}) 1.3$ antisense after spinal cord injury. J Neurophysiol 95:3343-3352.

11. Gerke MB, Duggan AW, Xu L, Siddall PJ (2003) Thalamic neuronal activity in rats with mechanical allodynia following contusive spinal cord injury. Neuroscience 117:715-722.

12. Huh Y, Cho J (2013) Discrete pattern of burst stimulation in the ventrobasal thalamus for anti-nociception. PLoS One 8:e67655.

13. Huh Y, Bhatt R, Jung D, Shin HS, Cho J (2012) Interactive responses of a thalamic neuron to formalin induced lasting pain in behaving mice. PLoS One 7:e30699.
14. Huh Y, Jung D, Seo T, Sun S, Kim SH, Rhim H, Chung S, Kim CH, Kwon Y, Bikson M, Chung YA, Kim JJ, Cho J (2018) Brain stimulation patterns emulating endogenous thalamocortical input to parvalbumin-expressing interneurons reduce nociception in mice. Brain Stimul 11:1151-1160.

15. Cerina M, Szkudlarek HJ, Coulon P, Meuth P, Kanyshkova T, Nguyen XV, Göbel K, Seidenbecher T, Meuth SG, Pape HC, Budde T (2015) Thalamic Kv 7 channels: pharmacological properties and activity control during noxious signal processing. Br J Pharmacol 172:3126-3140.

16. Hernandez O, Hernandez L, Vera D, Santander A, Zurek E (2015) Thalamic reticular cells firing modes and its dependency on the frequency and amplitude ranges of the current stimulus. Med Biol Eng Comput 53:37-44.

17. Destexhe A, Neubig M, Ulrich D, Huguenard J (1998) Dendritic low-threshold calcium currents in thalamic relay cells. J Neurosci 18:3574-3588.

18. Liu XB, Honda CN, Jones EG (1995) Distribution of four types of synapse on physiologically identified relay neurons in the ventral posterior thalamic nucleus of the cat. J Comp Neurol 352:69-91.

19. Hines ML, Carnevale NT (1997) The NEURON simulation environment. Neural Comput 9:1179-1209.

20. Llinás R, Jahnsen H (1982) Electrophysiology of mammalian thalamic neurones in vitro. Nature 297:406-408.

21. Edgerton JR, Jaeger D (2014) Optogenetic activation of nigral inhibitory inputs to motor thalamus in the mouse reveals classic inhibition with little potential for rebound activation. Front Cell Neurosci 8:36.

22. Netoff TI (2015) Spike time response curve. In: Encyclopedia of computational neuroscience (Jaeger D, Jung R, eds), pp 1-2. Springer Reference, New York, NY.

23. Park S, Kwag J (2012) Dendritic-targeting interneuron controls spike timing of hippocampal CA1 pyramidal neuron via activation of I(h). Neurosci Lett 523:9-14.

24. Destexhe A, Sejnowski TJ (2003) Interactions between membrane conductances underlying thalamocortical slow-wave oscillations. Physiol Rev 83:1401-1453.

25. Fuentealba P, Crochet S, Timofeev I, Bazhenov M, Sejnowski TJ, Steriade M (2004) Experimental evidence and modeling studies support a synchronizing role for electrical coupling in the cat thalamic reticular neurons in vivo. Eur J Neurosci 20:111-119.

26. Cox CL, Huguenard JR, Prince DA (1997) Nucleus reticularis neurons mediate diverse inhibitory effects in thalamus. Proc Natl Acad Sci U S A 94:8854-8859.

27. Huh Y, Cho J (2016) Differential responses of thalamic re- 
ticular neurons to nociception in freely behaving mice. Front Behav Neurosci 10:223.

28. Le Masson G, Renaud-Le Masson S, Debay D, Bal T (2002) Feedback inhibition controls spike transfer in hybrid thalamic circuits. Nature 417:854-858.

29. Kim YS, Yoon BE (2017) Altered GABAergic signaling in brain disease at various stages of life. Exp Neurobiol 26:122131.

30. Pita-Almenar JD, Yu D, Lu HC, Beierlein M (2014) Mechanisms underlying desynchronization of cholinergic-evoked thalamic network activity. J Neurosci 34:14463-14474.

31. LeBlanc BW, Cross B, Smith KA, Roach C, Xia J, Chao YC, Levitt J, Koyama S, Moore CI, Saab CY (2017) Thalamic bursts down-regulate cortical theta and nociceptive behavior. Sci Rep 7:2482.

32. Izhikevich EM (2002) Resonance and selective communication via bursts in neurons having subthreshold oscillations. Biosystems 67:95-102.

33. Izhikevich EM, Desai NS, Walcott EC, Hoppensteadt FC (2003) Bursts as a unit of neural information: selective com- munication via resonance. Trends Neurosci 26:161-167.

34. Naud R, Sprekeler H (2018) Sparse bursts optimize information transmission in a multiplexed neural code. Proc Natl Acad Sci U S A 115:E6329-E6338.

35. Mease RA, Kuner T, Fairhall AL, Groh A (2017) Multiplexed spike coding and adaptation in the thalamus. Cell Rep 19:1130-1140.

36. Ha GE, Cheong E (2017) Spike frequency adaptation in neurons of the central nervous system. Exp Neurobiol 26:179185.

37. Ha GE, Lee J, Kwak H, Song K, Kwon J, Jung SY, Hong J, Chang GE, Hwang EM, Shin HS, Lee CJ, Cheong E (2016) The Ca ${ }^{2+}$ activated chloride channel anoctamin-2 mediates spikefrequency adaptation and regulates sensory transmission in thalamocortical neurons. Nat Commun 7:13791.

38. Cheong E, Lee S, Choi BJ, Sun M, Lee CJ, Shin HS (2008) Tuning thalamic firing modes via simultaneous modulation of Tand L-type Ca2 + channels controls pain sensory gating in the thalamus. J Neurosci 28:13331-13340. 